\title{
Healthy and Unhealthy Leaf Classification using Convolution Neural Network and CSLBP Features
}

\author{
Harmandeep Kour, Lal Chand
}

\begin{abstract}
Once applied to real world images, most machine learning models for the automated identification of diseases have limited efficiency. Plant diseases cause major agricultural production and economic loss. These illnesses also show visible signs, including lines, streaks and shift in color, on leaf surfaces. Many researchers have recently researched the potential use of image treatment and computer processing in plants and leaves to diagnose disease. There is space for improved performance though several methods and computer procedures have been developed in this area of investigation. Several previous models only deal with a few morphological features of the diseased regions. A new method for detecting plant leave's disease using the segmentation, and CNN approach based on GLCM and LPQ features of the Basil and Guava leaves feedback imagery has been established in the present paper. The findings revealed that the suggested model is as effective as possible, for both basil and guava leaves, to better distinguish healthy and unhealthy leaves. The overall accuracy of the Guava dataset is $97.1 \%$ and the basil dataset is $\mathbf{9 2 . 1 \%}$.

Keywords: Bilateral Filter, CNN, GLCM, Leaf Disease Classification, LPQ.
\end{abstract}

\section{INTRODUCTION}

Throughout the numerous implementations, image analysis has its precious value. Agriculture is one of the most common fields of image processing. The need of agriculture is just not national economy and industrial areas. More than $50 \%$ of India's population is dependent on agriculture for their livelihood [10]. For detection of plant diseases, numerous fast methods need to be adopted. Sometimes the farmers are unable to identify the symptoms and apply the quantity of pesticides and fertilizers which result in the wrong effects on the plants as well as soil [16].

Revised Manuscript Received on September 15, 2020.

* Correspondence Author

Harmandeep Kour*, CSE, Punjabi University, Patiala, India. Email: caurharman07@gmail.com

Lal Chand Panwar, Assistant Professor, CSE, Punjabi University, patiala, India, Email: lc_panwar@yahoo.com

(C) The Authors. Published by Blue Eyes Intelligence Engineering and Sciences Publication (BEIESP). This is an open access article under the CC BY-NC-ND license (http://creativecommons.org/licenses/by-nc-nd/4.0/) limited to provide food but also it is a major contributor in

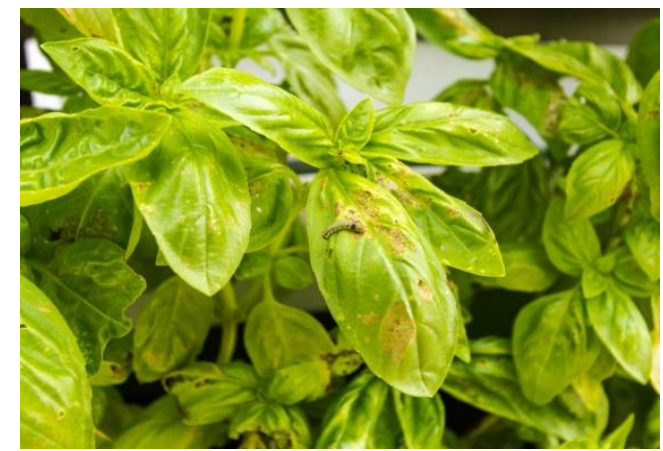

Figure 1: Disease of basil leaves (turns yellow and spot) Automation of the process of disease is the solution to this problem. Proper care of the crops is taken so that it doesn't cause any disease. Early detection of the plant diseases should be done so that the loss of the crops should be avoided. For example: a disease named "Little Leaf" found in Pine tree is in US. The affected tree stops growing and dies in early six years. In such cases, early detection could have been fruitful [26]. Some of the effective agriculture images processing areas are:

- Agriculture crops processing

- Plant and leaf processing

- Vegetable and fruit processing

- Soil and land processing [13].

It is much safer and simpler to diagnose automatically the diseases by having the signs on the plant leaves. The manual analysis of the large areas is an agitated method since time, energy and resources are required. Although it requires less energy to use robotic methods, less resources and tests are more accurate. The plants are easily monitored in automatic detection, and the symptoms on the plant leaves are detected automatically. This enables machine vision to automatically inspect, monitor and guide Robert on a picture-based basis [14]. Similarly, physically detecting illness needs a broad population and is less accurate. The main origin of leaf diseases is viral, fungal and bacterial [2]. Some diseases in Coconut trees, maize, papaya, chilly, cotton, brinjal, tomato are rust, yellow leaves disease, leaf rot, leaf curl, angular leave spot, leaves, late blight, and bacterial wilt [21]. Images of the multiple leaves are required and image processing methods and applied on the images to detect the diseases. Image filtering, Image segmentation, feature extraction; classification is done on the acquired images of the leaves to detect the diseases [19].

Published By: 
The leaf disease detection and classification of the leaves diseases is the key to prevent the agriculture loss [8].

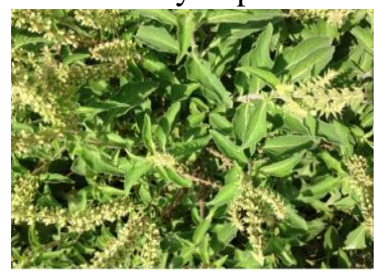

(a)

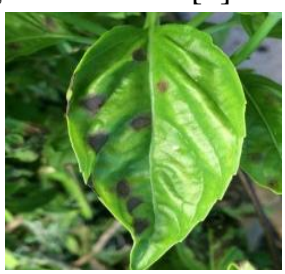

(b)

Figure 2: (a) Basil Leaf (b) Affected leaf

For conventional prescription medicines, leafs are the primary components. The WHO reports that about 80 percent of the world's population now depends on conventional drugs, much of which are plant-based medicines [25]. Also though researchers are working intensively to recognize plant leaf diseases utilizing different methods such as DNA / RNA, polymerase chain reaction, sensor techniques etc., however, computer vision tends to be less investigated for identifying the signs of diseases in medicinal plant leaf. The essay attempts to offer a method focused on the computer's perception for the stable or diseased identification of basil leaves. Basil, an ancient and common medicinal plant with essential micronutrients, is distinguished by health benefits. Throughout science and theology Basil has a tremendous meaning. High amounts (E)-beta-caryophyllene (BCP), which is expected to be effective in managing arthritis and inflammatory explosives, have been found inside the Swiss Federal Institute of Technology [3].

\section{RELATED WORK}

Bai et al. (2017) [4] developed a dynamic spot algorithm for extraction from a dynamic context of cucumber leaf conditions. The suggested algorithm offers the following developments and enhancements: (1) the goal leaf is effectively removed from context interferences with conflicting leaves and other non-target components by handling pictures with cucumber conditions against a complicated background using three runs of a specified water change algorithm. (2) The algorithm incorporates gray scale information for the area, which surmounts FCM's underuse of space picture pixel information and increases algorithm noise-filtering efficiency. (3) Improved outcomes are obtained by using rationally using space gray scale information using the weighted neighborhood gray values approach which refines cucumber spot edges.

Singh et al. (2019) [23] proposed a method to identifying and classifying diseases, it was effective to suggest a system of picture segmentation centered on psop for disease detection in the sunflower plantation leaf. No background knowledge is required as is the casual case for many current approaches as regards the amount of segments. Optimal tests were achieved with relatively little computational effort. This shows utility in detection and description of sunflower leaf conditions of the proposed algorithm. The findings of the tests indicate that few samples were misclassified. The accuracy rate of the proposed methodology is $98.0 \%$.

Wan, M. et al (2019) [9] Deep learning is a good way to identify tea leaf diseases, but a large number of training samples are required for training the profound learning

Published By:

Blue Eyes Intelligence Engineering and Sciences Publication

(C) Copyright: All rights reserved. model. It is impossible to obtain adequate samples of tea leaf diseases, because of limitations on realistic requirements. A low shot procedure for the diagnosis of the disease of tea leaves is suggested in this article. Spots of tea disease are separated from SVM photos of disease of tea leaf. C-DCGAN strengthens the disease spot samples; and the VGG 16 profound neural networks are equipped to recognize disease spots. Results from studies reveal that conventional machine learning approaches and methods of profound learning are successful in distinguishing tea leaves with complex histories. The overall precision of detection of tea red, tea violet and tea leaf location is $90 \%$ better than SVM, which is more than 30 percent.

Pandey, H. M. et al (2020) [27] designed compact CNN (Compact Net) software for identification and detection of leaf diseases was introduced. The research illustrated in the context current neural network architectures and explored their benefits and drawbacks in detail. A step by step review of the theoretical model has been provided so that other researchers can re-implement it for potential purposes. There are promising outcomes obtained for the emerging Dense Net. In determining three forms of maize leaf disease, $98.06 \%$ has been correctly classified.

Joshi, H. D. et al (2019). [7] Having effectively developed modern segmenting approaches with a new collection of features is the key focus of this article. All of the cycle from photos selection to segmentation and finally classification are defined respectively. New features were extracted on the basis of segmentation. For test the exactness of the suggested functions, nine classifiers are included. This model has been established to differentiate between stable leaves and diseases. Centered on the data presentation, RF performed with 98.4 percent precision better than other versions.

Agahi, H et al (2019). [15] The effect of wing entropy Tsallis in the fusion of photos with maximal reciprocal knowledge was observed. The findings may be merged into segmentation. Given that the estimation of cumulative shared knowledge is a time-consuming procedure in big data situations, a simple picture fusion approach is used. The best outcome is a quest inside the Tsallis entropy parameter space. In unattended segmentation of sheet pictures, the suggested approach helps overcoming the issue of complicated backgrounds and is not only restricted to leaf image segmentation. For extracting and processing the important information available in pixels of images, the image processing technology is used. The diseases can be detected from the input images through image processing. The training and testing are the two phases in which plant and leaf disease detection is performed. The training phase is the phase in which system can learn to detect the disease of the test set. The technique of feature extraction can be applied with the classification for plant and leaf diseases detection. In this research work, the approach of feature extraction and SVM classifier will be applied to detect the leaf disease.

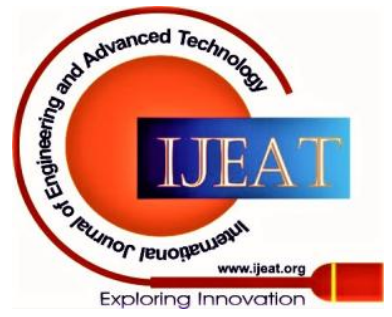

Expovation 


\section{SYSTEM ARCHITECTURE}

In this work, the implementation has been carried out in MATLAB. Basil Leaf dataset has been used for performance assessment of the proposed algorithm which classifies a given leaf into healthy or unhealthy category. The below flowchart gives description of steps followed in the present work.

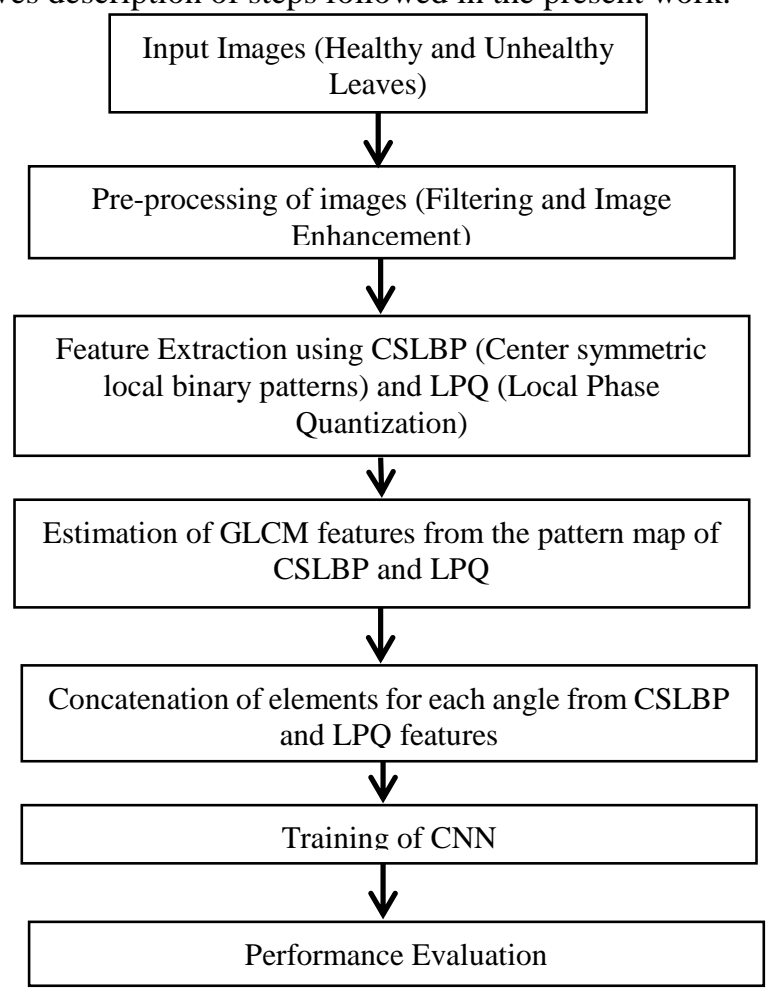

Figure 3: Feature extraction and training using CNN classifier

\section{A. Bilateral Filter}

In the first examples, Tomasi and Manduchi implemented bilateral filter[5] in 1998. When introduced in [20] was the idea of a bilateral filter as the SUSAN filter as a near-by filter. The Beltrami flow method is technically called the root of the bilateral filter, which generates a variety of techniques that improve picture from L2 linear diffusion to L1 nonlinear flows. The bilateral filter uses a weighted amount of the pixel values in the local area; both spatial length and intensity distance depend on weight. Therefore, the outlines of the vibration are well maintained.

\section{B. CSLBP (Center-Symmetric Local Binary Pattern)}

Central Symmetric Local Binary Pattern (CSLBP) is a modified version of the local binary pattern (LBP) algorithm. It is used to capture the texture and the gradient features of the image. This algorithm is computationally simple; has robustness on flat image areas as well as blurring and the illumination changes. CSLBP operator matches the grey level of the center symmetric couples of the pixel which represent the gradients of the image. The texture material is acquired by substituting each pixel in the image by the pixel's CSLBP value. The CSLBP can be defined as a modified version of the LBP (local binary pattern), as illustrated in Fig. 4.3 [24].

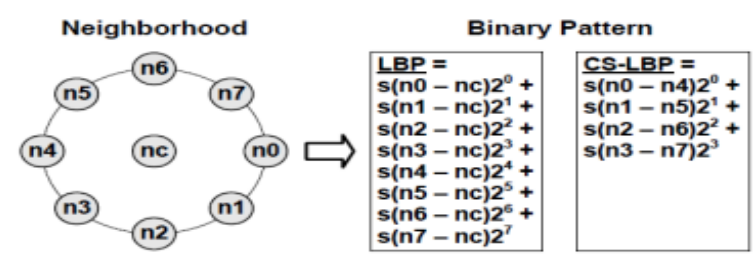

Figure 4: LBP and CSLBP descriptors for a neighborhood of $3 \times 3$ pixels [24]

Mathematically, the LBP is defined as:

$$
\begin{aligned}
& \operatorname{LBP}\left(x_{c}, y_{c}\right)=\sum_{n=0}^{n=7} s\left(g_{n}-g_{c}\right) 2^{n} \\
& s(x)=\left\{\begin{array}{l}
1, x \geq 0 \\
0, \text { otherwise }
\end{array}\right.
\end{aligned}
$$

where $g_{c}$ is the center pixel's gray value $\left(x_{c}, y_{c}\right)$, and $g_{n}$ represents 8 neighboring pixels. If $g_{n}$ is lesser than $g_{c}$, then the result of pixel is set to 0 ; otherwise, it is fixed to 1 . In CSLBP, instead of comparing the neighboring pixels with the center pixel, the center-symmetric pairs of pixels, such as (g0, g4), (g1, g5), (g2, g6), and (g3, g7), are computed as follows [24]:

$\operatorname{CSLBP}\left(x_{c}, y_{c}\right)=\sum_{n=0}^{n=3} s\left(g_{n}-g_{n+4}\right) 2^{n}$

$s(x)=\left\{\begin{array}{l}1, x \geq 0 \\ 0, \text { otherwise }\end{array}\right.$

\section{C.C. Convolutional Neural Network}

CNNs represent a special type of neural network called as Convolutional Neural networks (CNNs). Outputs to the Input Layer are passed to hidden layers, point operations are performed (multiplication and addition) by the corresponding weights and prejudices for each node and output values are generated by the activation method. The basic operating concept is the same for CNNs or the regular neural networks. Network parameters are determined in the output nodes and modified after each iteration of the training information using the back-propagation principle. In fact, because of increased difficulty, CNNs are more challenging to train than other shallow neural networks. CNNs benefit from any spatial correlation input structure, such as images, so that the CNN arranges neurons in the spatial dimensions. This space method facilitates effective knowledge transfer through the network and decreases the amount of network parameters significantly. In other words, spatially related information is used in the CNN structure. CNNs can define phenomena and recognize them more reliably and efficiently by adopting this theory. A CNN has just a few simple components, convolution layer, pooling layer, relu layer and completely/full connected layer. It is the major component of its design.

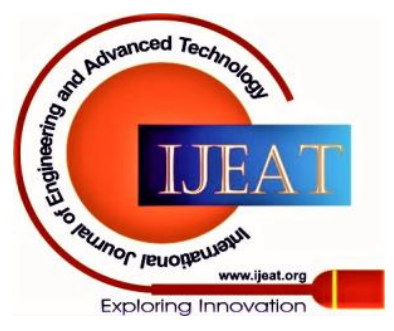




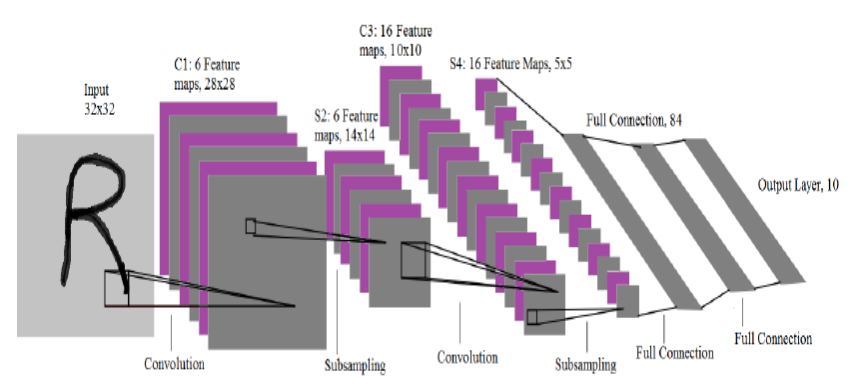

Figure 5: Architecture of LeNet-5, a convolutional neural network for digit recognition [28].

\section{Leaf images dataset}

The dataset of rice leaf is obtained from the UCI repository of dataset

(https://archive.ics.uci.edu/ml/datasets/Rice+Leaf+Diseases\# ). It contains the three classes of leaf disease namely Bacterial leaf blight, Brown spot, and Leaf smut each with 40 images. The dataset taken from these classes for training and testing of CNN is in the ratio of 0.7 and 0.3 (70\% for training and 30\% for testing). During our experiments, we used Matlab (2015a) to measure the performance of the classification process, defined as:

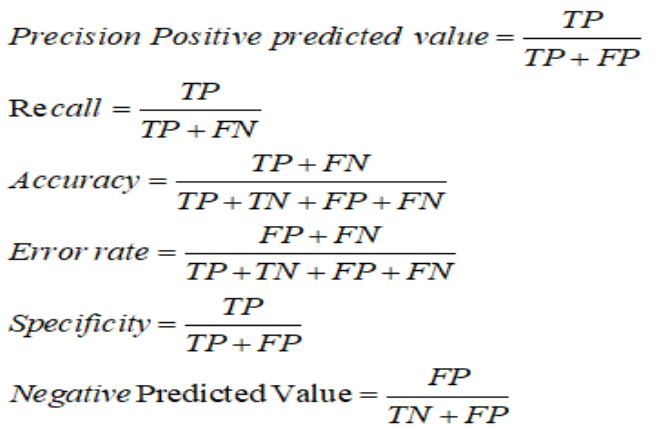

Where, TP represents the value of True Positive, TN represents the value of True Negative, FP represents the value of False Positive, FN represents the value of False Negative

\section{RESULTS AND DISCUSSIONS}

Two datasets of leaf namely Basil and Guava leaves are considered in this research work to test the proposed technique. The Guava dataset is taken from the online available database "A Database of Leaf Images: Practice towards Plant Conservation with Plant Pathology" [1] containing 419 images (277 healthy images and 142 unhealthy images). The dataset of basil leaves is collected manually containing 64 images (32 healthy leaves and 32 unhealthy leaves). For training the CNN, 70\% ratio of dataset is considered and for testing all the images of both dataset is considered. Below table 1 shows the results for Guava leaves having healthy and unhealthy categories.

TABLE 1: Pre-Processing results for Guava (healthy and unhealthy leaves)

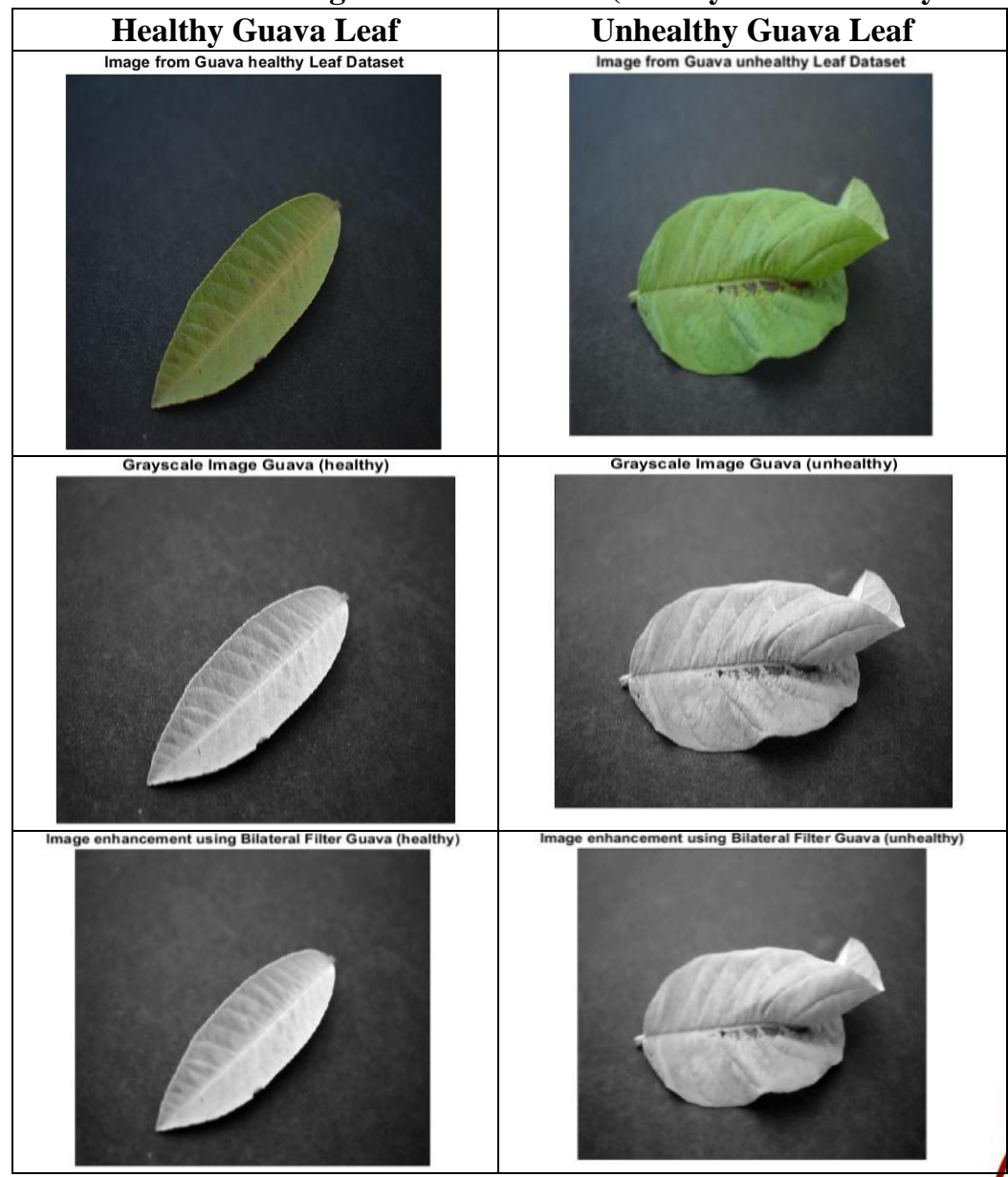

Published By:

Blue Eyes Intelligence Engineering and Sciences Publication

(C) Copyright: All rights reserved.
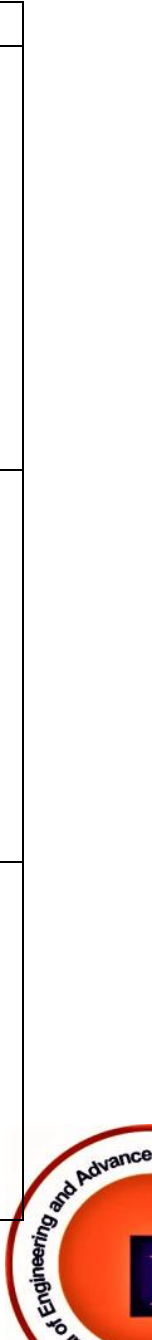


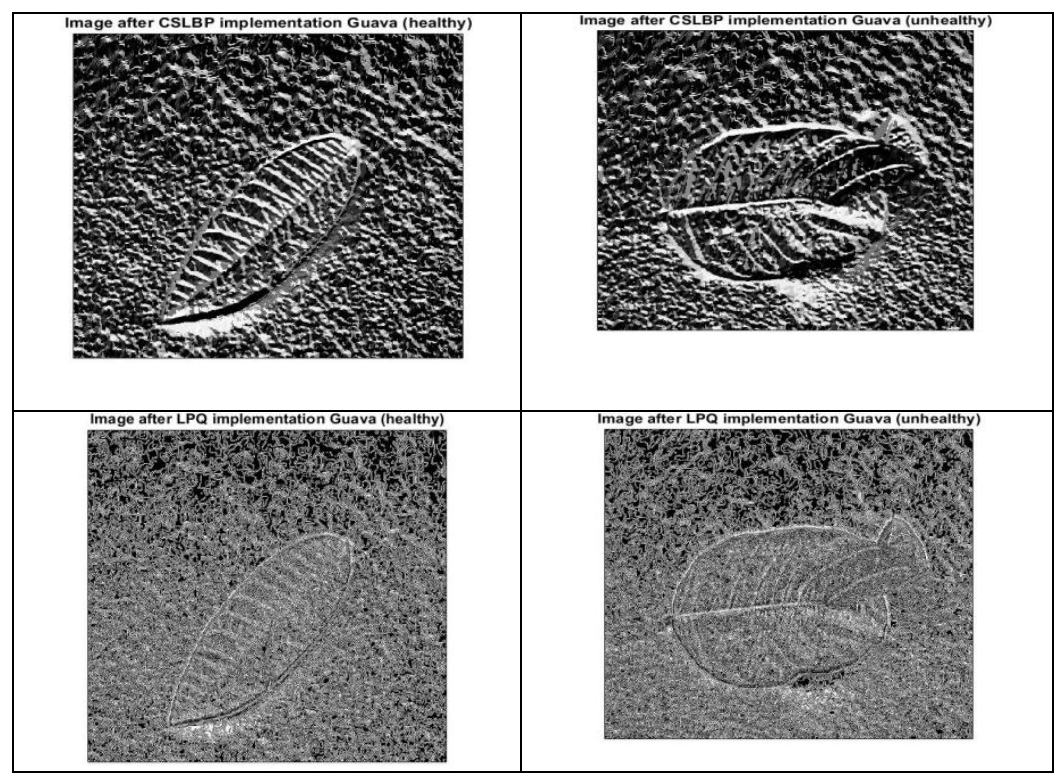

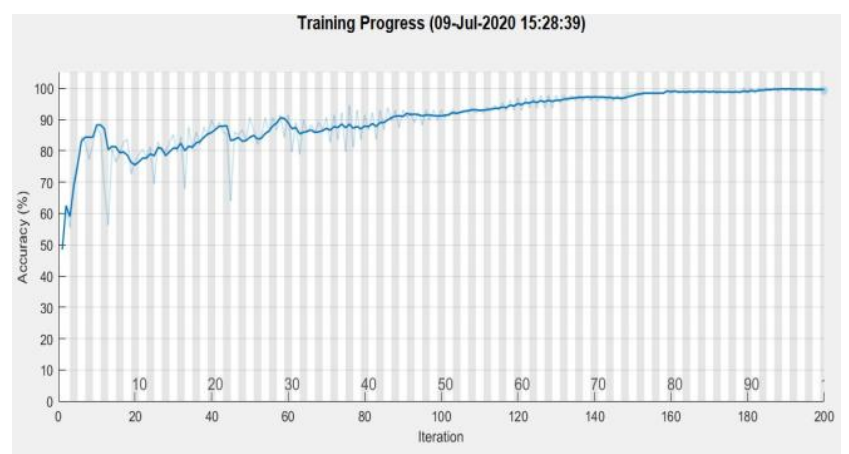

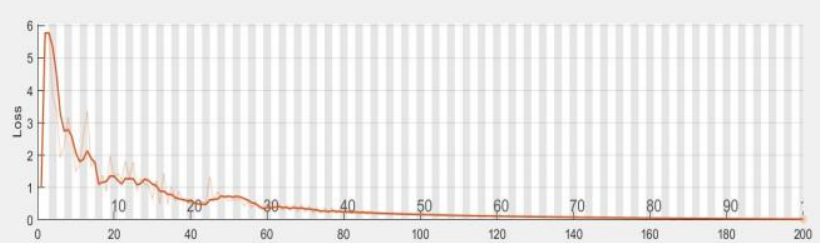

Figure 6: Training Accuracy for Guava leaves

Fig. 6 show the images of network training in Matlab. It can be seen that the training accuracy is $100 \%$ for and loss is $0 \%$ for guava leaf dataset.

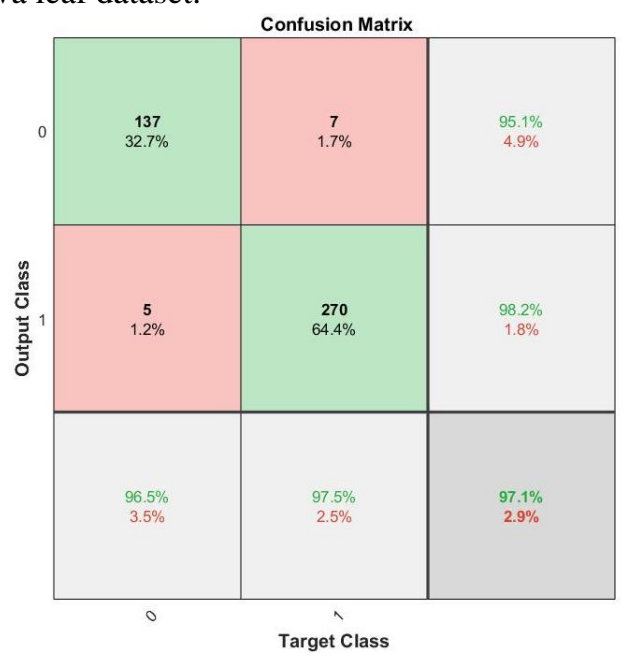

Figure 7: Confusion testing on trained CNN Matrix for Guava leaf

The fig. 7 shows the confusion matrix obtained after the testing of all the images of guava leaf category. The trained network to identify the healthy and unhealthy guava leaves achieves the accuracy of $97.1 \%$. Similarly, the second dataset of Bail leaf is also trained and tested using the CNN and similar results were obtained. The performance metrics achieved for both the datasets using CNN are shown in next fig.

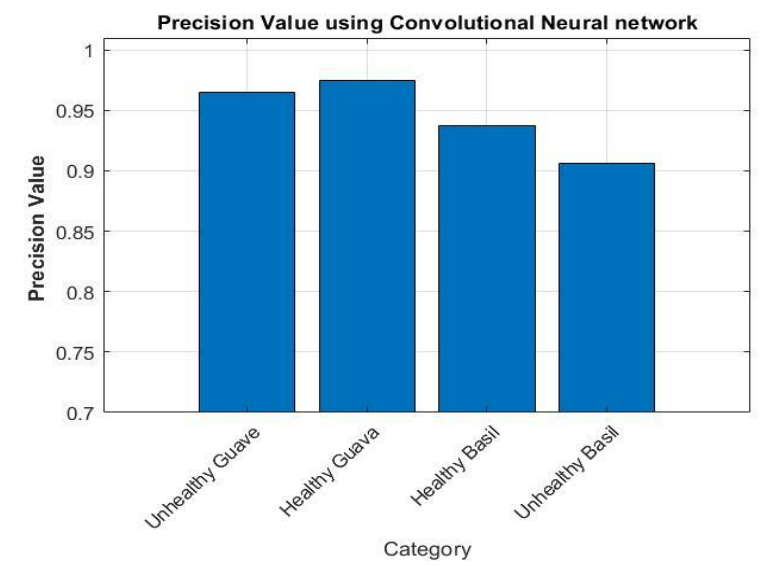

Figure 8: Precision value for all the categories of leaves using CNN

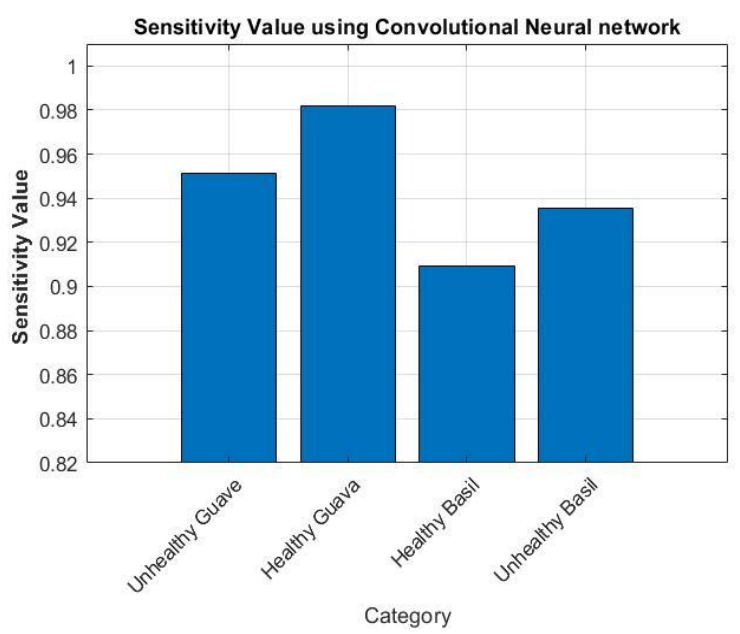

Figure 9: Sensitivity value for all the categories of leaves using CNN

Published By:

Blue Eyes Intelligence Engineering

and Sciences Publication

(C) Copyright: All rights reserved.

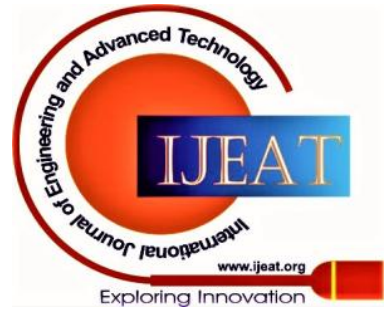




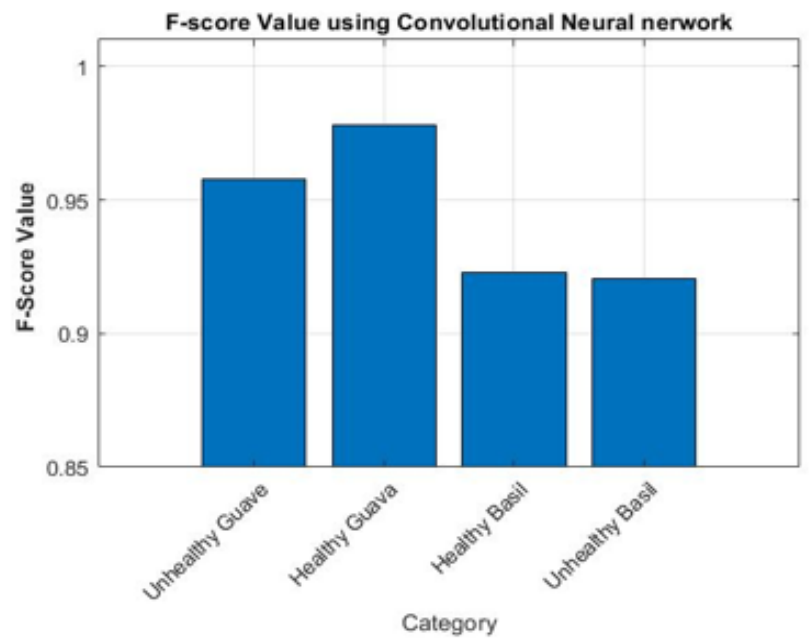

Figure 10: F-score value for all the categories of leaves using CNN

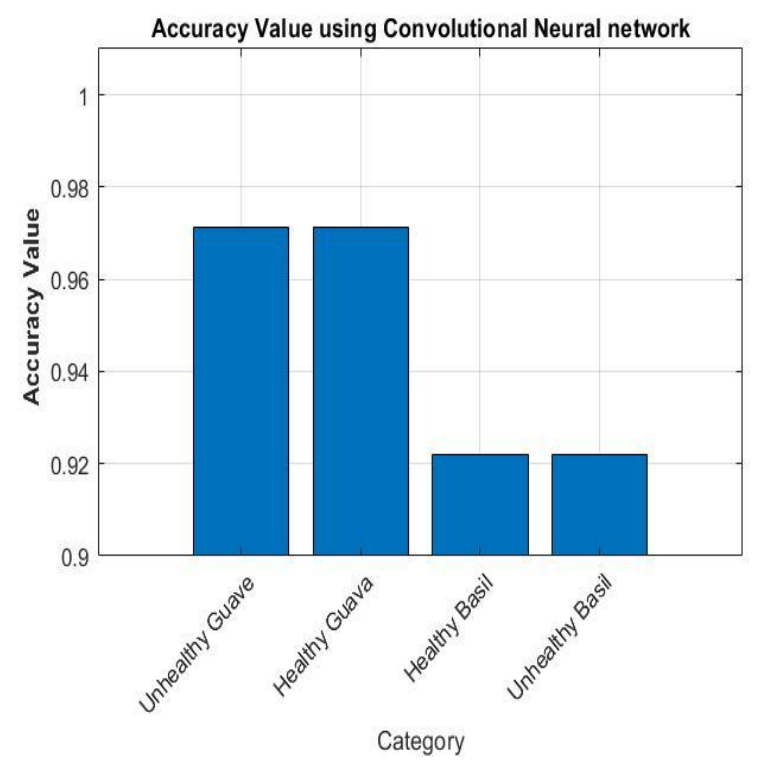

Figure 11: Accuracy value for all the categories of leaves using CNN

The fig. 8 to 11 show results of precision, sensitivity, f-score, and accuracy respectively. These shows the performance of the trained CNN in categorizing the healthy and unhealthy leaves of Guava and Basil. It can be seen that the CNN of Guava dataset achieved 97.1\% accuracy whereas the basil CNN achieves $92.1 \%$ accuracy that shows the efficient performance of the proposed network architecture.

\section{CONCLUSION}

Once applied to real world images, most machine learning models for the automated identification of diseases have limited efficiency. Plant diseases cause major agricultural production and economic loss. These illnesses also show visible signs, including lines, streaks and shift in color, on leaf surfaces. In order to classify the disease, the proliferation of insect diseases has contributed to the advancement of imaging and machine learning methods. Recently several researchers have thoroughly investigated the possible use of image processing and machine learning for detection of disease in plants and leaves. While having developed many methods and computer algorithms in this field of study, there is still scope for improved achievements. Some preceding models discuss only a few morphological features of the diseased regions. In the present work, a new approach has been developed to detect the disease of plant leaves by using segmentation, and CNN approach based on GLCM and LPQ features of the input images for Basil and Guava leaves. From the results, it has been found that the proposed model is efficient enough to detect the healthy and unhealthy leaves correctly for both the basil and guava leaves dataset. The overall accuracy found is 97.1\% for Guava dataset and $92.1 \%$ for basil dataset.

\section{REFRENCES}

1. A Database of Leaf Images: Practice towards Plant Conservation with Plant Pathology Available at: http://dx.doi.org/10.17632/hb74ynkjcn.4\#folder-d2a758f3-cac5-4a2a -8c9f-90efbd0df308

2. A Gargade, A., \& Khandekar, M. S. (2019). A Review: Custard Apple Leaf Parameter Analysis and Leaf Disease Detection using Digital Image Processing. In 2019 3rd International Conference on Computing Methodologies and Communication (ICCMC) (pp. 267-271). IEEE.

3. Izzo, A. A., Borrelli, F., Capasso, R., Di Marzo, V., \& Mechoulam, R. (2009). Non-psychotropic plant cannabinoids: new therapeutic opportunities from an ancient herb. Trends in pharmacological sciences, 30(10), 515-527.

4. Bai, X., Li, X., Fu, Z., Lv, X., \& Zhang, L. (2017). A fuzzy clustering segmentation method based on neighborhood grayscale information for defining cucumber leaf spot disease images. Computers and Electronics in Agriculture, 136, 157-165.

5. Tomasi, C., \& Manduchi, R. (1998). Bilateral filtering for gray and color images. In Sixth international conference on computer vision (IEEE Cat. No. 98CH36271) (pp. 839-846). IEEE.

6. Dhall, A., Asthana, A., Goecke, R., \& Gedeon, T. (2011, March) Emotion recognition using PHOG and LPQ features. In Face and Gesture 2011 (pp. 878-883). IEEE.

7. Dhingra, G., Kumar, V., \& Joshi, H. D. (2019). A novel computer vision based neutrosophic approach for leaf disease identification and classification. Measurement, 135, 782-794.

8. Kambale, G., \& Bilgi, D. N. (2017). A Survey Paper On Crop Disease Identification And Classification Using Pattern Recognition And Digital Image Processing Techniques.

9. Hu, G., Wu, H., Zhang, Y., \& Wan, M. (2019). A low shot learning method for tea leaf's disease identification. Computers and Electronics in Agriculture, 163, 104852.

10. Kumar, K. V., \& Jayasankar, T. (2019). An identification of crop disease using image segmentation. Int. J. Pharm. Sci. Res, 10(3), 1054-1064.

11. Khan, M. A., Akram, T., Sharif, M., Awais, M., Javed, K., Ali, H., \& Saba, T. (2018). CCDF: Automatic system for segmentation and recognition of fruit crops diseases based on correlation coefficient and deep CNN features. Computers and electronics in agriculture, 155, 220-236.

12. Kumar, J. P., \& Domnic, S. (2019). Image based leaf segmentation and counting in rosette plants. Information Processing In Agriculture, 6(2), 233-246.

13. Chahal, N. (2015). A study on agricultural image processing along with classification model. In 2015 IEEE International Advance Computing Conference (IACC) (pp. 942-947). IEEE.

14. Dhaygude, S. B., \& Kumbhar, N. P. (2013). Agricultural plant leaf disease detection using image processing. International Journal of Advanced Research in Electrical, Electronics and Instrumentation Engineering, 2(1), 599-602.

15. Nikbakhsh, N., Baleghi, Y., \& Agahi, H. (2019). Maximum mutual information and Tsallis entropy for unsupervised segmentation of tree leaves in natural scenes. Computers and Electronics in Agriculture, 162, 440-449.

Published By:

Blue Eyes Intelligence Engineering

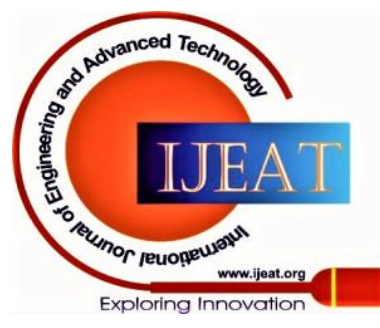


16. Anand, R., Veni, S., \& Aravinth, J. (2016, April). An application of image processing techniques for detection of diseases on brinjal leaves using k-means clustering method. In 2016 international conference on recent trends in information technology (ICRTIT) (pp. 1-6). IEEE.

17. Haralick, R. M., Shanmugam, K., \& Dinstein, I. H. (1973). Textural features for image classification. IEEE Transactions on systems, man, and cybernetics, (6), 610-621.

18. Rico-Fernández, M. P., Rios-Cabrera, R., Castelán, M., Guerrero-Reyes, H. I., \& Juarez-Maldonado, A. (2019). A contextualized approach for segmentation of foliage in different crop species. Computers and Electronics in Agriculture, 156, 378-386.

19. Khirade, S. D., \& Patil, A. B. (2015). Plant disease detection using image processing. In 2015 International conference on computing communication control and automation (pp. 768-771). IEEE.

20. Smith, S. M., \& Brady, J. M. (1997). SUSAN - a new approach to low level image processing. International journal of computer vision, 23(1), 45-78.

21. Raghavendra, B. K. (2019). Diseases Detection of Various Plant Leaf Using Image Processing Techniques: A Review. In 2019 5th International Conference on Advanced Computing \& Communication Systems (ICACCS) (pp. 313-316). IEEE.

22. Saleem, G., Akhtar, M., Ahmed, N., \& Qureshi, W. S. (2019) Automated analysis of visual leaf shape features for plant classification. Computers and Electronics in Agriculture, 157, 270-280.

23. Singh, V. (2019). Sunflower leaf diseases detection using image segmentation based on particle swarm optimization. Artificial Intelligence in Agriculture, 3, 62-68.

24. Uliyan, D. M., Jalab, H. A., \& Wahab, A. W. A. (2015). Copy move image forgery detection using Hessian and center symmetric local binary pattern. In 2015 IEEE Conference on Open Systems (ICOS) (pp. 7-11). IEEE.

25. Varshney, P., \& Suresh, S. Integration of organic farming practices in cultivation of Ayurveda herbs: An innovative approach.

26. Singh, V., \& Misra, A. K. (2017). Detection of plant leaf diseases using image segmentation and soft computing techniques. Information processing in Agriculture, 4(1), 41-49.

27. Waheed, A., Goyal, M., Gupta, D., Khanna, A., Hassanien, A. E., \& Pandey, H. M. (2020). An optimized dense convolutional neura network model for disease recognition and classification in corn leaf. Computers and Electronics in Agriculture, 175, 105456.

28. LeCun, Y., Bottou, L., Bengio, Y., \& Haffner, P. (1998). Gradient-based learning applied to document recognition. Proceedings of the IEEE, 86(11), 2278-2324.

\section{AUTHORS PROFILE}

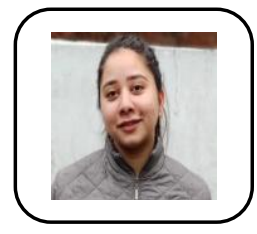

Harmandeep Kaur, received B.Tech degree in Computer Science and Engineering from Chandigarh Group Of Colleges, Landran, India, in May 2014. She is pursuing M.Tech in Computer Science and Engineering at Punjabi University, Patiala, India. Area of Interest: Digital Image Processing.

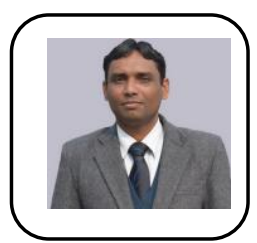

Lal Chand Panwar, has received his B.Tech Degree in Computer Science And Engineering from NIT, Jaipur and his M.Tech Degree in Computer Science and Engineering from Punjabi University, Patiala, India. He is pursuing his PHD in Computer Science and engineering from Punjabi University, Patiala. He is Working as a Assistant Professor in CSE department of Punjabi University, Patiala.. He has Achieved a Tremendous Amount of Publications Ranging to 27. His area of interest is Network Security.

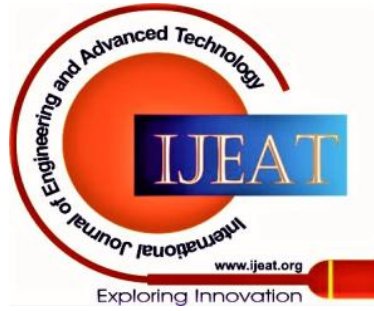

